\title{
Guessing, Mind-Changing, and the Second Ambiguous Class
}

\author{
Samuel Alexander
}

\begin{abstract}
In his dissertation, Wadge defined a notion of guessability on subsets of the Baire space and gave two characterizations of guessable sets. A set is guessable if and only if it is in the second ambiguous class $\left(\Delta_{2}^{0}\right)$, if and only if it is eventually annihilated by a certain remainder. We simplify this remainder and give a new proof of the latter equivalence. We then introduce a notion of guessing with an ordinal limit on how often one can change one's mind. We show that for every ordinal $\alpha$, a guessable set is annihilated by $\alpha$ applications of the simplified remainder if and only if it is guessable with fewer than $\alpha$ mind changes. We use guessability with fewer than $\alpha$ mind changes to give a semicharacterization of the Hausdorff difference hierarchy, and indicate how Wadge's notion of guessability can be generalized to higher-order guessability, providing characterizations of $\Delta_{\alpha}^{0}$ for all successor ordinals $\alpha>1$.
\end{abstract}

\section{Introduction}

Let $\mathbb{N}^{\mathbb{N}}$ be the set of sequences $s: \mathbb{N} \rightarrow \mathbb{N}$, and let $\mathbb{N}<\mathbb{N}$ be the set $\bigcup_{n} \mathbb{N}^{n}$ of finite sequences. If $s \in \mathbb{N}^{<\mathbb{N}}$, we will write $[s]$ for $\left\{f \in \mathbb{N}^{\mathbb{N}}: f\right.$ extends $\left.s\right\}$. We equip $\mathbb{N}^{\mathbb{N}}$ with a second-countable topology by declaring $[s]$ to be a basic open set whenever $s \in \mathbb{N}^{<\mathbb{N}}$.

Throughout the paper, $S$ will denote a subset of $\mathbb{N}^{\mathbb{N}}$. We say that $S \in \Delta_{2}^{0}$ if $S$ is simultaneously a countable intersection of open sets and a countable union of closed sets in the above topology. In classic terminology, $S \in \Delta_{2}^{0}$ just in case $S$ is both $G_{\delta}$ and $F_{\sigma}$.

The following notion was discovered by Wadge [9, pp. 141-42] and independently by this author [1, Section 2, Definition 1, p. 2]. ${ }^{1}$

Received September 3, 2013; accepted January 8, 2014

First published online January 6, 2016

2010 Mathematics Subject Classification: Primary 03E15

Keywords: guessability, difference hierarchy, descriptive hierarchy

(C) 2016 by University of Notre Dame 10.1215/00294527-3443549 


\title{
Guessing, Mind-Changing, and the Second Ambiguous Class
}

\author{
Samuel Alexander
}

\begin{abstract}
In his dissertation, Wadge defined a notion of guessability on subsets of the Baire space and gave two characterizations of guessable sets. A set is guessable if and only if it is in the second ambiguous class $\left(\Delta_{2}^{0}\right)$, if and only if it is eventually annihilated by a certain remainder. We simplify this remainder and give a new proof of the latter equivalence. We then introduce a notion of guessing with an ordinal limit on how often one can change one's mind. We show that for every ordinal $\alpha$, a guessable set is annihilated by $\alpha$ applications of the simplified remainder if and only if it is guessable with fewer than $\alpha$ mind changes. We use guessability with fewer than $\alpha$ mind changes to give a semicharacterization of the Hausdorff difference hierarchy, and indicate how Wadge's notion of guessability can be generalized to higher-order guessability, providing characterizations of $\Delta_{\alpha}^{0}$ for all successor ordinals $\alpha>1$.
\end{abstract}

\section{Introduction}

Let $\mathbb{N}^{\mathbb{N}}$ be the set of sequences $s: \mathbb{N} \rightarrow \mathbb{N}$, and let $\mathbb{N}<\mathbb{N}$ be the set $\bigcup_{n} \mathbb{N}^{n}$ of finite sequences. If $s \in \mathbb{N}^{<\mathbb{N}}$, we will write $[s]$ for $\left\{f \in \mathbb{N}^{\mathbb{N}}: f\right.$ extends $\left.s\right\}$. We equip $\mathbb{N}^{\mathbb{N}}$ with a second-countable topology by declaring $[s]$ to be a basic open set whenever $s \in \mathbb{N}^{<\mathbb{N}}$.

Throughout the paper, $S$ will denote a subset of $\mathbb{N}^{\mathbb{N}}$. We say that $S \in \Delta_{2}^{0}$ if $S$ is simultaneously a countable intersection of open sets and a countable union of closed sets in the above topology. In classic terminology, $S \in \Delta_{2}^{0}$ just in case $S$ is both $G_{\delta}$ and $F_{\sigma}$.

The following notion was discovered by Wadge [9, pp. 141-42] and independently by this author [1, Section 2, Definition 1, p. 2]. ${ }^{1}$

Received September 3, 2013; accepted January 8, 2014

First published online January 6, 2016

2010 Mathematics Subject Classification: Primary 03E15

Keywords: guessability, difference hierarchy, descriptive hierarchy

(C) 2016 by University of Notre Dame 10.1215/00294527-3443549 


\title{
Guessing, Mind-Changing, and the Second Ambiguous Class
}

\author{
Samuel Alexander
}

\begin{abstract}
In his dissertation, Wadge defined a notion of guessability on subsets of the Baire space and gave two characterizations of guessable sets. A set is guessable if and only if it is in the second ambiguous class $\left(\Delta_{2}^{0}\right)$, if and only if it is eventually annihilated by a certain remainder. We simplify this remainder and give a new proof of the latter equivalence. We then introduce a notion of guessing with an ordinal limit on how often one can change one's mind. We show that for every ordinal $\alpha$, a guessable set is annihilated by $\alpha$ applications of the simplified remainder if and only if it is guessable with fewer than $\alpha$ mind changes. We use guessability with fewer than $\alpha$ mind changes to give a semicharacterization of the Hausdorff difference hierarchy, and indicate how Wadge's notion of guessability can be generalized to higher-order guessability, providing characterizations of $\Delta_{\alpha}^{0}$ for all successor ordinals $\alpha>1$.
\end{abstract}

\section{Introduction}

Let $\mathbb{N}^{\mathbb{N}}$ be the set of sequences $s: \mathbb{N} \rightarrow \mathbb{N}$, and let $\mathbb{N}<\mathbb{N}$ be the set $\bigcup_{n} \mathbb{N}^{n}$ of finite sequences. If $s \in \mathbb{N}^{<\mathbb{N}}$, we will write $[s]$ for $\left\{f \in \mathbb{N}^{\mathbb{N}}: f\right.$ extends $\left.s\right\}$. We equip $\mathbb{N}^{\mathbb{N}}$ with a second-countable topology by declaring $[s]$ to be a basic open set whenever $s \in \mathbb{N}^{<\mathbb{N}}$.

Throughout the paper, $S$ will denote a subset of $\mathbb{N}^{\mathbb{N}}$. We say that $S \in \Delta_{2}^{0}$ if $S$ is simultaneously a countable intersection of open sets and a countable union of closed sets in the above topology. In classic terminology, $S \in \Delta_{2}^{0}$ just in case $S$ is both $G_{\delta}$ and $F_{\sigma}$.

The following notion was discovered by Wadge [9, pp. 141-42] and independently by this author [1, Section 2, Definition 1, p. 2]. ${ }^{1}$

Received September 3, 2013; accepted January 8, 2014

First published online January 6, 2016

2010 Mathematics Subject Classification: Primary 03E15

Keywords: guessability, difference hierarchy, descriptive hierarchy

(C) 2016 by University of Notre Dame 10.1215/00294527-3443549 


\title{
Guessing, Mind-Changing, and the Second Ambiguous Class
}

\author{
Samuel Alexander
}

\begin{abstract}
In his dissertation, Wadge defined a notion of guessability on subsets of the Baire space and gave two characterizations of guessable sets. A set is guessable if and only if it is in the second ambiguous class $\left(\Delta_{2}^{0}\right)$, if and only if it is eventually annihilated by a certain remainder. We simplify this remainder and give a new proof of the latter equivalence. We then introduce a notion of guessing with an ordinal limit on how often one can change one's mind. We show that for every ordinal $\alpha$, a guessable set is annihilated by $\alpha$ applications of the simplified remainder if and only if it is guessable with fewer than $\alpha$ mind changes. We use guessability with fewer than $\alpha$ mind changes to give a semicharacterization of the Hausdorff difference hierarchy, and indicate how Wadge's notion of guessability can be generalized to higher-order guessability, providing characterizations of $\Delta_{\alpha}^{0}$ for all successor ordinals $\alpha>1$.
\end{abstract}

\section{Introduction}

Let $\mathbb{N}^{\mathbb{N}}$ be the set of sequences $s: \mathbb{N} \rightarrow \mathbb{N}$, and let $\mathbb{N}<\mathbb{N}$ be the set $\bigcup_{n} \mathbb{N}^{n}$ of finite sequences. If $s \in \mathbb{N}^{<\mathbb{N}}$, we will write $[s]$ for $\left\{f \in \mathbb{N}^{\mathbb{N}}: f\right.$ extends $\left.s\right\}$. We equip $\mathbb{N}^{\mathbb{N}}$ with a second-countable topology by declaring $[s]$ to be a basic open set whenever $s \in \mathbb{N}^{<\mathbb{N}}$.

Throughout the paper, $S$ will denote a subset of $\mathbb{N}^{\mathbb{N}}$. We say that $S \in \Delta_{2}^{0}$ if $S$ is simultaneously a countable intersection of open sets and a countable union of closed sets in the above topology. In classic terminology, $S \in \Delta_{2}^{0}$ just in case $S$ is both $G_{\delta}$ and $F_{\sigma}$.

The following notion was discovered by Wadge [9, pp. 141-42] and independently by this author [1, Section 2, Definition 1, p. 2]. ${ }^{1}$

Received September 3, 2013; accepted January 8, 2014

First published online January 6, 2016

2010 Mathematics Subject Classification: Primary 03E15

Keywords: guessability, difference hierarchy, descriptive hierarchy

(C) 2016 by University of Notre Dame 10.1215/00294527-3443549 


\title{
Guessing, Mind-Changing, and the Second Ambiguous Class
}

\author{
Samuel Alexander
}

\begin{abstract}
In his dissertation, Wadge defined a notion of guessability on subsets of the Baire space and gave two characterizations of guessable sets. A set is guessable if and only if it is in the second ambiguous class $\left(\Delta_{2}^{0}\right)$, if and only if it is eventually annihilated by a certain remainder. We simplify this remainder and give a new proof of the latter equivalence. We then introduce a notion of guessing with an ordinal limit on how often one can change one's mind. We show that for every ordinal $\alpha$, a guessable set is annihilated by $\alpha$ applications of the simplified remainder if and only if it is guessable with fewer than $\alpha$ mind changes. We use guessability with fewer than $\alpha$ mind changes to give a semicharacterization of the Hausdorff difference hierarchy, and indicate how Wadge's notion of guessability can be generalized to higher-order guessability, providing characterizations of $\Delta_{\alpha}^{0}$ for all successor ordinals $\alpha>1$.
\end{abstract}

\section{Introduction}

Let $\mathbb{N}^{\mathbb{N}}$ be the set of sequences $s: \mathbb{N} \rightarrow \mathbb{N}$, and let $\mathbb{N}<\mathbb{N}$ be the set $\bigcup_{n} \mathbb{N}^{n}$ of finite sequences. If $s \in \mathbb{N}^{<\mathbb{N}}$, we will write $[s]$ for $\left\{f \in \mathbb{N}^{\mathbb{N}}: f\right.$ extends $\left.s\right\}$. We equip $\mathbb{N}^{\mathbb{N}}$ with a second-countable topology by declaring $[s]$ to be a basic open set whenever $s \in \mathbb{N}^{<\mathbb{N}}$.

Throughout the paper, $S$ will denote a subset of $\mathbb{N}^{\mathbb{N}}$. We say that $S \in \Delta_{2}^{0}$ if $S$ is simultaneously a countable intersection of open sets and a countable union of closed sets in the above topology. In classic terminology, $S \in \Delta_{2}^{0}$ just in case $S$ is both $G_{\delta}$ and $F_{\sigma}$.

The following notion was discovered by Wadge [9, pp. 141-42] and independently by this author [1, Section 2, Definition 1, p. 2]. ${ }^{1}$

Received September 3, 2013; accepted January 8, 2014

First published online January 6, 2016

2010 Mathematics Subject Classification: Primary 03E15

Keywords: guessability, difference hierarchy, descriptive hierarchy

(C) 2016 by University of Notre Dame 10.1215/00294527-3443549 


\title{
Guessing, Mind-Changing, and the Second Ambiguous Class
}

\author{
Samuel Alexander
}

\begin{abstract}
In his dissertation, Wadge defined a notion of guessability on subsets of the Baire space and gave two characterizations of guessable sets. A set is guessable if and only if it is in the second ambiguous class $\left(\Delta_{2}^{0}\right)$, if and only if it is eventually annihilated by a certain remainder. We simplify this remainder and give a new proof of the latter equivalence. We then introduce a notion of guessing with an ordinal limit on how often one can change one's mind. We show that for every ordinal $\alpha$, a guessable set is annihilated by $\alpha$ applications of the simplified remainder if and only if it is guessable with fewer than $\alpha$ mind changes. We use guessability with fewer than $\alpha$ mind changes to give a semicharacterization of the Hausdorff difference hierarchy, and indicate how Wadge's notion of guessability can be generalized to higher-order guessability, providing characterizations of $\Delta_{\alpha}^{0}$ for all successor ordinals $\alpha>1$.
\end{abstract}

\section{Introduction}

Let $\mathbb{N}^{\mathbb{N}}$ be the set of sequences $s: \mathbb{N} \rightarrow \mathbb{N}$, and let $\mathbb{N}<\mathbb{N}$ be the set $\bigcup_{n} \mathbb{N}^{n}$ of finite sequences. If $s \in \mathbb{N}^{<\mathbb{N}}$, we will write $[s]$ for $\left\{f \in \mathbb{N}^{\mathbb{N}}: f\right.$ extends $\left.s\right\}$. We equip $\mathbb{N}^{\mathbb{N}}$ with a second-countable topology by declaring $[s]$ to be a basic open set whenever $s \in \mathbb{N}^{<\mathbb{N}}$.

Throughout the paper, $S$ will denote a subset of $\mathbb{N}^{\mathbb{N}}$. We say that $S \in \Delta_{2}^{0}$ if $S$ is simultaneously a countable intersection of open sets and a countable union of closed sets in the above topology. In classic terminology, $S \in \Delta_{2}^{0}$ just in case $S$ is both $G_{\delta}$ and $F_{\sigma}$.

The following notion was discovered by Wadge [9, pp. 141-42] and independently by this author [1, Section 2, Definition 1, p. 2]. ${ }^{1}$

Received September 3, 2013; accepted January 8, 2014

First published online January 6, 2016

2010 Mathematics Subject Classification: Primary 03E15

Keywords: guessability, difference hierarchy, descriptive hierarchy

(C) 2016 by University of Notre Dame 10.1215/00294527-3443549 


\title{
Guessing, Mind-Changing, and the Second Ambiguous Class
}

\author{
Samuel Alexander
}

\begin{abstract}
In his dissertation, Wadge defined a notion of guessability on subsets of the Baire space and gave two characterizations of guessable sets. A set is guessable if and only if it is in the second ambiguous class $\left(\Delta_{2}^{0}\right)$, if and only if it is eventually annihilated by a certain remainder. We simplify this remainder and give a new proof of the latter equivalence. We then introduce a notion of guessing with an ordinal limit on how often one can change one's mind. We show that for every ordinal $\alpha$, a guessable set is annihilated by $\alpha$ applications of the simplified remainder if and only if it is guessable with fewer than $\alpha$ mind changes. We use guessability with fewer than $\alpha$ mind changes to give a semicharacterization of the Hausdorff difference hierarchy, and indicate how Wadge's notion of guessability can be generalized to higher-order guessability, providing characterizations of $\Delta_{\alpha}^{0}$ for all successor ordinals $\alpha>1$.
\end{abstract}

\section{Introduction}

Let $\mathbb{N}^{\mathbb{N}}$ be the set of sequences $s: \mathbb{N} \rightarrow \mathbb{N}$, and let $\mathbb{N}<\mathbb{N}$ be the set $\bigcup_{n} \mathbb{N}^{n}$ of finite sequences. If $s \in \mathbb{N}^{<\mathbb{N}}$, we will write $[s]$ for $\left\{f \in \mathbb{N}^{\mathbb{N}}: f\right.$ extends $\left.s\right\}$. We equip $\mathbb{N}^{\mathbb{N}}$ with a second-countable topology by declaring $[s]$ to be a basic open set whenever $s \in \mathbb{N}^{<\mathbb{N}}$.

Throughout the paper, $S$ will denote a subset of $\mathbb{N}^{\mathbb{N}}$. We say that $S \in \Delta_{2}^{0}$ if $S$ is simultaneously a countable intersection of open sets and a countable union of closed sets in the above topology. In classic terminology, $S \in \Delta_{2}^{0}$ just in case $S$ is both $G_{\delta}$ and $F_{\sigma}$.

The following notion was discovered by Wadge [9, pp. 141-42] and independently by this author [1, Section 2, Definition 1, p. 2]. ${ }^{1}$

Received September 3, 2013; accepted January 8, 2014

First published online January 6, 2016

2010 Mathematics Subject Classification: Primary 03E15

Keywords: guessability, difference hierarchy, descriptive hierarchy

(C) 2016 by University of Notre Dame 10.1215/00294527-3443549 


\title{
Guessing, Mind-Changing, and the Second Ambiguous Class
}

\author{
Samuel Alexander
}

\begin{abstract}
In his dissertation, Wadge defined a notion of guessability on subsets of the Baire space and gave two characterizations of guessable sets. A set is guessable if and only if it is in the second ambiguous class $\left(\Delta_{2}^{0}\right)$, if and only if it is eventually annihilated by a certain remainder. We simplify this remainder and give a new proof of the latter equivalence. We then introduce a notion of guessing with an ordinal limit on how often one can change one's mind. We show that for every ordinal $\alpha$, a guessable set is annihilated by $\alpha$ applications of the simplified remainder if and only if it is guessable with fewer than $\alpha$ mind changes. We use guessability with fewer than $\alpha$ mind changes to give a semicharacterization of the Hausdorff difference hierarchy, and indicate how Wadge's notion of guessability can be generalized to higher-order guessability, providing characterizations of $\Delta_{\alpha}^{0}$ for all successor ordinals $\alpha>1$.
\end{abstract}

\section{Introduction}

Let $\mathbb{N}^{\mathbb{N}}$ be the set of sequences $s: \mathbb{N} \rightarrow \mathbb{N}$, and let $\mathbb{N}<\mathbb{N}$ be the set $\bigcup_{n} \mathbb{N}^{n}$ of finite sequences. If $s \in \mathbb{N}^{<\mathbb{N}}$, we will write $[s]$ for $\left\{f \in \mathbb{N}^{\mathbb{N}}: f\right.$ extends $\left.s\right\}$. We equip $\mathbb{N}^{\mathbb{N}}$ with a second-countable topology by declaring $[s]$ to be a basic open set whenever $s \in \mathbb{N}^{<\mathbb{N}}$.

Throughout the paper, $S$ will denote a subset of $\mathbb{N}^{\mathbb{N}}$. We say that $S \in \Delta_{2}^{0}$ if $S$ is simultaneously a countable intersection of open sets and a countable union of closed sets in the above topology. In classic terminology, $S \in \Delta_{2}^{0}$ just in case $S$ is both $G_{\delta}$ and $F_{\sigma}$.

The following notion was discovered by Wadge [9, pp. 141-42] and independently by this author [1, Section 2, Definition 1, p. 2]. ${ }^{1}$

Received September 3, 2013; accepted January 8, 2014

First published online January 6, 2016

2010 Mathematics Subject Classification: Primary 03E15

Keywords: guessability, difference hierarchy, descriptive hierarchy

(C) 2016 by University of Notre Dame 10.1215/00294527-3443549 


\title{
Guessing, Mind-Changing, and the Second Ambiguous Class
}

\author{
Samuel Alexander
}

\begin{abstract}
In his dissertation, Wadge defined a notion of guessability on subsets of the Baire space and gave two characterizations of guessable sets. A set is guessable if and only if it is in the second ambiguous class $\left(\Delta_{2}^{0}\right)$, if and only if it is eventually annihilated by a certain remainder. We simplify this remainder and give a new proof of the latter equivalence. We then introduce a notion of guessing with an ordinal limit on how often one can change one's mind. We show that for every ordinal $\alpha$, a guessable set is annihilated by $\alpha$ applications of the simplified remainder if and only if it is guessable with fewer than $\alpha$ mind changes. We use guessability with fewer than $\alpha$ mind changes to give a semicharacterization of the Hausdorff difference hierarchy, and indicate how Wadge's notion of guessability can be generalized to higher-order guessability, providing characterizations of $\Delta_{\alpha}^{0}$ for all successor ordinals $\alpha>1$.
\end{abstract}

\section{Introduction}

Let $\mathbb{N}^{\mathbb{N}}$ be the set of sequences $s: \mathbb{N} \rightarrow \mathbb{N}$, and let $\mathbb{N}<\mathbb{N}$ be the set $\bigcup_{n} \mathbb{N}^{n}$ of finite sequences. If $s \in \mathbb{N}^{<\mathbb{N}}$, we will write $[s]$ for $\left\{f \in \mathbb{N}^{\mathbb{N}}: f\right.$ extends $\left.s\right\}$. We equip $\mathbb{N}^{\mathbb{N}}$ with a second-countable topology by declaring $[s]$ to be a basic open set whenever $s \in \mathbb{N}^{<\mathbb{N}}$.

Throughout the paper, $S$ will denote a subset of $\mathbb{N}^{\mathbb{N}}$. We say that $S \in \Delta_{2}^{0}$ if $S$ is simultaneously a countable intersection of open sets and a countable union of closed sets in the above topology. In classic terminology, $S \in \Delta_{2}^{0}$ just in case $S$ is both $G_{\delta}$ and $F_{\sigma}$.

The following notion was discovered by Wadge [9, pp. 141-42] and independently by this author [1, Section 2, Definition 1, p. 2]. ${ }^{1}$

Received September 3, 2013; accepted January 8, 2014

First published online January 6, 2016

2010 Mathematics Subject Classification: Primary 03E15

Keywords: guessability, difference hierarchy, descriptive hierarchy

(C) 2016 by University of Notre Dame 10.1215/00294527-3443549 


\title{
Guessing, Mind-Changing, and the Second Ambiguous Class
}

\author{
Samuel Alexander
}

\begin{abstract}
In his dissertation, Wadge defined a notion of guessability on subsets of the Baire space and gave two characterizations of guessable sets. A set is guessable if and only if it is in the second ambiguous class $\left(\Delta_{2}^{0}\right)$, if and only if it is eventually annihilated by a certain remainder. We simplify this remainder and give a new proof of the latter equivalence. We then introduce a notion of guessing with an ordinal limit on how often one can change one's mind. We show that for every ordinal $\alpha$, a guessable set is annihilated by $\alpha$ applications of the simplified remainder if and only if it is guessable with fewer than $\alpha$ mind changes. We use guessability with fewer than $\alpha$ mind changes to give a semicharacterization of the Hausdorff difference hierarchy, and indicate how Wadge's notion of guessability can be generalized to higher-order guessability, providing characterizations of $\Delta_{\alpha}^{0}$ for all successor ordinals $\alpha>1$.
\end{abstract}

\section{Introduction}

Let $\mathbb{N}^{\mathbb{N}}$ be the set of sequences $s: \mathbb{N} \rightarrow \mathbb{N}$, and let $\mathbb{N}<\mathbb{N}$ be the set $\bigcup_{n} \mathbb{N}^{n}$ of finite sequences. If $s \in \mathbb{N}^{<\mathbb{N}}$, we will write $[s]$ for $\left\{f \in \mathbb{N}^{\mathbb{N}}: f\right.$ extends $\left.s\right\}$. We equip $\mathbb{N}^{\mathbb{N}}$ with a second-countable topology by declaring $[s]$ to be a basic open set whenever $s \in \mathbb{N}^{<\mathbb{N}}$.

Throughout the paper, $S$ will denote a subset of $\mathbb{N}^{\mathbb{N}}$. We say that $S \in \Delta_{2}^{0}$ if $S$ is simultaneously a countable intersection of open sets and a countable union of closed sets in the above topology. In classic terminology, $S \in \Delta_{2}^{0}$ just in case $S$ is both $G_{\delta}$ and $F_{\sigma}$.

The following notion was discovered by Wadge [9, pp. 141-42] and independently by this author [1, Section 2, Definition 1, p. 2]. ${ }^{1}$

Received September 3, 2013; accepted January 8, 2014

First published online January 6, 2016

2010 Mathematics Subject Classification: Primary 03E15

Keywords: guessability, difference hierarchy, descriptive hierarchy

(C) 2016 by University of Notre Dame 10.1215/00294527-3443549 


\title{
Guessing, Mind-Changing, and the Second Ambiguous Class
}

\author{
Samuel Alexander
}

\begin{abstract}
In his dissertation, Wadge defined a notion of guessability on subsets of the Baire space and gave two characterizations of guessable sets. A set is guessable if and only if it is in the second ambiguous class $\left(\Delta_{2}^{0}\right)$, if and only if it is eventually annihilated by a certain remainder. We simplify this remainder and give a new proof of the latter equivalence. We then introduce a notion of guessing with an ordinal limit on how often one can change one's mind. We show that for every ordinal $\alpha$, a guessable set is annihilated by $\alpha$ applications of the simplified remainder if and only if it is guessable with fewer than $\alpha$ mind changes. We use guessability with fewer than $\alpha$ mind changes to give a semicharacterization of the Hausdorff difference hierarchy, and indicate how Wadge's notion of guessability can be generalized to higher-order guessability, providing characterizations of $\Delta_{\alpha}^{0}$ for all successor ordinals $\alpha>1$.
\end{abstract}

\section{Introduction}

Let $\mathbb{N}^{\mathbb{N}}$ be the set of sequences $s: \mathbb{N} \rightarrow \mathbb{N}$, and let $\mathbb{N}<\mathbb{N}$ be the set $\bigcup_{n} \mathbb{N}^{n}$ of finite sequences. If $s \in \mathbb{N}^{<\mathbb{N}}$, we will write $[s]$ for $\left\{f \in \mathbb{N}^{\mathbb{N}}: f\right.$ extends $\left.s\right\}$. We equip $\mathbb{N}^{\mathbb{N}}$ with a second-countable topology by declaring $[s]$ to be a basic open set whenever $s \in \mathbb{N}^{<\mathbb{N}}$.

Throughout the paper, $S$ will denote a subset of $\mathbb{N}^{\mathbb{N}}$. We say that $S \in \Delta_{2}^{0}$ if $S$ is simultaneously a countable intersection of open sets and a countable union of closed sets in the above topology. In classic terminology, $S \in \Delta_{2}^{0}$ just in case $S$ is both $G_{\delta}$ and $F_{\sigma}$.

The following notion was discovered by Wadge [9, pp. 141-42] and independently by this author [1, Section 2, Definition 1, p. 2]. ${ }^{1}$

Received September 3, 2013; accepted January 8, 2014

First published online January 6, 2016

2010 Mathematics Subject Classification: Primary 03E15

Keywords: guessability, difference hierarchy, descriptive hierarchy

(C) 2016 by University of Notre Dame 10.1215/00294527-3443549 


\title{
Guessing, Mind-Changing, and the Second Ambiguous Class
}

\author{
Samuel Alexander
}

\begin{abstract}
In his dissertation, Wadge defined a notion of guessability on subsets of the Baire space and gave two characterizations of guessable sets. A set is guessable if and only if it is in the second ambiguous class $\left(\Delta_{2}^{0}\right)$, if and only if it is eventually annihilated by a certain remainder. We simplify this remainder and give a new proof of the latter equivalence. We then introduce a notion of guessing with an ordinal limit on how often one can change one's mind. We show that for every ordinal $\alpha$, a guessable set is annihilated by $\alpha$ applications of the simplified remainder if and only if it is guessable with fewer than $\alpha$ mind changes. We use guessability with fewer than $\alpha$ mind changes to give a semicharacterization of the Hausdorff difference hierarchy, and indicate how Wadge's notion of guessability can be generalized to higher-order guessability, providing characterizations of $\Delta_{\alpha}^{0}$ for all successor ordinals $\alpha>1$.
\end{abstract}

\section{Introduction}

Let $\mathbb{N}^{\mathbb{N}}$ be the set of sequences $s: \mathbb{N} \rightarrow \mathbb{N}$, and let $\mathbb{N}<\mathbb{N}$ be the set $\bigcup_{n} \mathbb{N}^{n}$ of finite sequences. If $s \in \mathbb{N}^{<\mathbb{N}}$, we will write $[s]$ for $\left\{f \in \mathbb{N}^{\mathbb{N}}: f\right.$ extends $\left.s\right\}$. We equip $\mathbb{N}^{\mathbb{N}}$ with a second-countable topology by declaring $[s]$ to be a basic open set whenever $s \in \mathbb{N}^{<\mathbb{N}}$.

Throughout the paper, $S$ will denote a subset of $\mathbb{N}^{\mathbb{N}}$. We say that $S \in \Delta_{2}^{0}$ if $S$ is simultaneously a countable intersection of open sets and a countable union of closed sets in the above topology. In classic terminology, $S \in \Delta_{2}^{0}$ just in case $S$ is both $G_{\delta}$ and $F_{\sigma}$.

The following notion was discovered by Wadge [9, pp. 141-42] and independently by this author [1, Section 2, Definition 1, p. 2]. ${ }^{1}$

Received September 3, 2013; accepted January 8, 2014

First published online January 6, 2016

2010 Mathematics Subject Classification: Primary 03E15

Keywords: guessability, difference hierarchy, descriptive hierarchy

(C) 2016 by University of Notre Dame 10.1215/00294527-3443549 